Article

\title{
Evaluating Auction Mechanisms for the Preservation of Cost-Aware Digital Objects under Constrained Digital Preservation Budgets
}

\author{
Andres El-Fakdi $*, t, \pm\left(\mathbb{D}\right.$ and Josep Lluis de la Rosa ${ }^{t, \ddagger(\mathbb{D}}$ \\ TECNIO Centre EASY, VICOROB Institute, University of Girona, 17071 Girona, Spain; peplluis@eia.udg.edu \\ * Correspondence: aelfakdi@eia.udg.edu \\ + Current address: Department of Electrical Engineering, Electronics and Automation (EEEA), Campus Montilivi, \\ Polytechnic School of the University of Girona, Building P4, 17071 Girona, Spain. \\ $\ddagger$ These authors contributed equally to this work.
}

check for

updates

Citation: El-Fakdi, A.; de la Rosa, J.L. Evaluating Auction Mechanisms for the Preservation of Cost-Aware Digital Objects under Constrained Digital Preservation Budgets. Mathematics 2022, 10, 92. https:// doi.org/10.3390/math10010092

Academic Editor: Radi Romansky

Received: 2 December 2021

Accepted: 23 December 2021

Published: 28 December 2021

Publisher's Note: MDPI stays neutral with regard to jurisdictional claims in published maps and institutional affiliations.

Copyright: (c) 2021 by the authors. Licensee MDPI, Basel, Switzerland. This article is an open access article distributed under the terms and conditions of the Creative Commons Attribution (CC BY) license (https:/ / creativecommons.org/licenses/by/ $4.0 /)$.

\begin{abstract}
Digital preservation is a field of research focused on designing strategies for maintaining digital objects accessible for general use in the coming years. Out of the many approaches to digital preservation, the present research article is a continuation work of a previously published article containing a proposal for a novel object-centered paradigm to address the digital preservation problem where digital objects share part of the responsibility for self-preservation. In the new framework, the behavior of digital objects is modeled to find the best preservation strategy. The results presented in the current article add a new economic constraint to the object behavior. Now, differently from the previous paper, migrations, copies and updates are not free to use, but subject to budget limitations to ensure the economic sustainability of the whole preservation system, forcing the now-called cost-aware digital objects for efficient management of available budget. The presented approach compares two auction-based mechanisms, a multi-unit auction and a combinatorial auction, with a simple direct purchase strategy as possible efficient behaviors for budget management. TiM, a simulated environment for running distributed digital ecosystems, is used to perform the experiments. The simulated results map the relation between the studied purchase models with the sustained quality level of digital objects, as a measure of its accessibility, together with its budget management capabilities. About the results, the best performance corresponds to the combinatorial auction model. The results are a good approach to deal with the digital preservation problem from a sustainable point of view and open the door to future implementations with other purchase strategies.
\end{abstract}

Keywords: digital preservation; distributed architectures; self-preserving digital objects

\section{Introduction}

The need for research into new ways of preserving digital content over the long term has been evident for over the last three decades. Examples of important digital data loss are numerous. In the early 1980s, the National Aeronautics and Space Administration (NASA) lost track of high-quality footage of the Apollo 11 moonwalk because backup tapes were lost or recorded over. As the real-time broadcast worked and was widely recorded, preservation of the backup video was not deemed a priority in the years immediately following the mission [1]. Another example is the Newham Museum Archaelogical Service in the UK that lost ten years of fieldwork on about 230 floppy disks containing over 6000 files due to technology obsolescence. The files were in a variety of proprietary formats that are now archaic, and some of digital materials stored in these files have become inaccessible [2]. Long-term digital preservation (LTDP), or simply digital preservation (DP), is an increasing focus of businesses and public sector agencies, as well as scientists and citizens. The challenge in preserving valuable digital information-consisting of text, music, images, multimedia documents, web pages, sensor data, 3D design models, or more 
recently like NFT (Non-Fungible Tokens), digital assets, etc., generated throughout all areas of our society-is real and growing at an exponential pace. Preservation is not only about making files accessible, but also to protect them, making security and privacy two major concerns associated with digital object preservation [3]. The preservation problem is, however, not limited to cultural, economic, historical, administrative and scientific information. Legislation is placing an increasingly large burden on commercial, industrial, cultural and governmental organizations to ensure that their digital records are retained, made accessible, and effectively preserved in the long term. Therefore, in the recent years, memory institutions and academic organizations have taken the lead in defining solutions for addressing LTDP issues. As far as the PLANETS project [4] in the late 2000s estimated that the value of digital documents in danger of digital obsolescence produced only in the European Union was at the time more than $€ 3$ billion files per year. At the international scale, a study by the International Data Corporation (IDC) concluded that stored information volumes are reaching huge proportions and will become impossible to manage in a short period of time [5]. Keeping this pace up, the risk of a massive data loss due to a future unexpected chain of events or critical software and hardware obsolescence is real. These studies also point out that the costs for addressing knowledge preservation are enormous and, in order to prevent major losses in the long term, research must be focused on designing efficient systems [6,7].

It is widely accepted that digitalized information has many advantages over old printed information: easy to duplicate, easy to move, and easy to keep. On the other hand, digital data needs a continuous stream of energy for its maintenance, and it suffers a unique longevity concern that printed copies do not: short life-cycle across changing file formats due to software adoption waves [8]. Centralized storage systems share some advantages: maximization of data integrity, minimization of redundancies, easier security requirements and maintenance services, among others. They also have major drawbacks: connectivity dependence, high traffic bottlenecks or critical hardware failures being the most serious ones [9]. Nowadays, LTDP focus is shifting away from large-scale digital top-down archiving systems toward distributed, object-centered approaches, making digital objects and the information they contain the center of research and study [10,11]. By definition, LTDP mechanisms are uncertain and bound to unknown future technology changes, so it makes sense to program them as intelligent objects with learning abilities. In this line, successful results proposed in [11-13] leave a path open for digital objects to incorporate intelligent behaviors designed for self-preservation. This change of paradigm, from machine-to-human intelligence to machine-to-machine intelligence, imports concepts from computational ecologies [14] and defines the conceptual term of self-preserving digital object (SPDO). Thus, if the previous work presented in [15] explored how computational intelligence can model digital object behaviors in order to obtain the best preservation strategy, the work proposed in this article goes one step further and adds one more constrain into the self-preservation equation: the long term economical sustainability of SPDOs, where the SPDOs spend a given budget efficiently searching for the best preservation services. Avoiding future data deluge will have a cost, and it makes sense that, in a distributed environment where intelligent digital objects are programmed to autonomously find the best preservation strategies, part of this intelligence is dedicated to assure optimized costs and high efficiency at budget management. Experts conclude that digital preservation is about creating a data economy in which those who care, those who pay, and those who preserve are working in coordination. Sustainability concerns on the long-term preservation of digital objects frequently focuses on the difficulty of technical issues, leaving the organizational and economic issues less articulated. However, these are implicit in the notion of sustainability, which, at the risk of oversimplification, comes down to two questions: How much does it cost and, is it the most affordable cost? In addition, there is a much more difficult question: Who is going to pay for the preservation efforts? Horizontally distributed knowledge architectures should democratize knowledge, making it available 
to everybody. In the same way, cost burdens should be distributed proportionally among institutions, governments and global society.

This article aims to introduce budget management capabilities inside the intelligent SPDO paradigm. We think that part of the self-preservation success is about the economic sustainability of the whole preservation system and the correct management of the budget that digital objects have for their preservation, thus becoming active actors in their own LTDP management. This concept generates a new definition inside the SPDO paradigm: the cost-aware digital object (CADO). A CADO is a digital, intelligently programmed object, which uses a particular budget to negotiate with digital preservation services present in the Internet, looking for format migration, extraction of metadata or new storage in search of the most cost-efficient preservation solution. Negotiation can be defined as an interaction of influences [14]. Examples of such interactions include the process of resolving disputes, agreeing upon courses of action, bargaining for individual or collective advantage, or crafting outcomes to satisfy various interests of two or more parties. Negotiation contains a process involving dealing and communication among the parties, which intend to reach a mutually accepted agreement on a given matter and commit to a course of action [16]. Depending on the focus of the process, a negotiation can be classified as a quantitative or qualitative negotiation process. The main form of quantitative negotiations, i.e., auctions, focuses on price negotiation. Auctions follow a clearly structured procedure, for example Dutch or English auction, according to which competitive bids are placed until an agreement is reached. On the other hand, qualitative negotiations do not have the competitive approach, but are based on a more unstructured dealing and bargaining negotiation process. Furthermore, negotiation processes can also be classified according to the number of participants and the number of aspects that are under consideration. With respect to number of participants, bilateral and multi-party negotiation can be distinguished, whereas single and multi-issue negotiations can be identified as considered aspects in a negotiation. The more parties and aspects involved, the more complex the negotiation process becomes.

In this article, authors focus on quantitative auctions as the defined negotiation protocol. CADOs, as active agents involved with their preservation, focus on the price of the preservation services they bid for. Thus, the authors' proposal focuses on the managing of the budget of SPDOs through object-to-service negotiations by an auctionbased mechanism system. Thus, the article simulates three simple electronic auction mechanisms and analyzes the results obtained, comparing them to find the best costefficient solution. The objectives of the article are:

- The proposal of a new auction-based cost-aware sense for programming intelligent self-sustained digital objects inside the SPDO paradigm for the solution of the digital preservation problem. The proposed approach describes an environment where intelligent digital objects are programmed with cost-aware capabilities and budget management. Thus, SPDOs manage specific budget resources available in a distributed network to guarantee their survival in the long-term, negotiating with storing repositories and web services in order to find the best cost-efficient self-preservation strategy;

- The article investigates the performance of auction-based mechanisms as an initial approach for developing cost-aware self-preserving behaviors for digital objects. Features present in auction-based methodologies make them good candidates to be used for optimizing SPDO economic efficiency and open the door to future experimentation with other AI-based algorithms for preservation cost management;

- In order to demonstrate the feasibility of the proposed auction-based cost-aware paradigm and the programmed auction mechanisms, a simulated environment has been designed to test and compare the performance offered by the three different purchase systems: a direct purchase method and two auction-based mechanisms.

The article is structured as follows: Section 2 details the current digital preservation platforms and the former work related to cost-aware digital preservation strategies. Section 3 gives a detailed explanation of the simulation environment and the actors involved: the cost-aware self-preserved digital object, CADO, inside the SPDO paradigm, the 
preservation services and the network description. Section 4 describes the main features of an electronic auction and presents the three auction-based methods selected to carry out the simulated experiments; all three methodologies used for comparison are well-known auction mechanisms from the literature and offer a solid benchmark for results analysis. Section 5 presents the simulation software platform developed for the tests. Section 6 provides the simulated results obtained during the experiments. Section 7 discusses the results obtained and comparatively analyzes the performance of the methods, and finally, Section 8 concludes this study and addresses future research directions.

\section{Background and Related Work}

Current initiatives related to DP are centralized, top-to-bottom structures mainly focused on solving issues related with standardization of file formats and technical properties of digital objects needed for correct migrations and updates. Such services offer multiple storage features, and all implement the standard OAIS preservation model [17] and the Dublin Core metadata standard [18]. Following these standards, we find institutions like the International Federation of Library Associations and Institutions (IFLA) [19] or the Digital Preservation Program carried out by UNESCO [20]. Additionally, open-source initiatives have proved successful into securing these standards, the best known examples being the Repository of Authentic Digital Objects (RODA) [21], the Dynamic Digital Repository (DSpace) [22], the Flexible and Extensible Digital Object Repository Architecture (FEDORA) [23], and EPrints Institutional Repository Software [24]. On the other side of the architecture design map, both commercial and open source decentralized repositories are starting to gain visibility driven by the surge of blockchain technologies and decentralized structures. Good examples of it are Filecoin [25], Storj [26], and Sia [27]. All these architectures, both centralized and decentralized, implement the previously mentioned standards to ensure preservation and rely on the use of a set of widely accepted descriptors, organized into categories: provenance, authenticity, preservation activity, technical environment, or rights management. In turn, none of them take into account parameters related to the preservation cost or consider any kind of cost-efficient preservation factor as a part of their preservation strategy.

In the 1990s, researchers and technologists started to point out their main concerns about costs related to future needed storage capabilities to match digital preservation needs [28]. Changes in the DP terminology, misunderstandings of meanings and a lack of direct business planning gave a wrong scenario where the DP community fought the problem of technological obsolescence without sustained support from organizations that supposedly need it the most. Organizations care about ensuring their continued existence and profitability. Some years later, consensus started to shift from how to overcome technological barriers to how to overcome with the maintenance costs to guarantee the preservation in the long term. As stated in [29], a lack of money is the biggest obstacle to effective DP as assumptions that make digital preservation more expensive reduce the likelihood of it happening at all. Early attempts to model preservation costs of digital objects were mainly oriented towards aspects only related to the technological cost of the preservation (the Dutch Testbed project [30]). Such approaches were later superseded by a more holistic view (eSPIDA framework [31]) and empirical life cycle studies (the LIFE, LIFE2 and LIFE3 projects [32]). These projects based their models on large memory institutions and developed methods for calculating the cost of running a digital repository or maintaining a digital object throughout its life span. The work of the Blue Ribbon Task Force on sustainable digital preservation and access [33] was a significant addition to the literature on digital preservation and represented the first systematic attempt to focus not just on the cost of managing information over time, but on the economic framework that is required to allow that to occur. One of their central statements concludes that ensuring digital assets to be available for future use is not simply a matter of finding sufficient funds, it is about mobilizing resources-human, technical, and financial-across a spectrum of stakeholders diffused over both space and time. Economic sustainability of digital preser- 
vation was further elaborated in the UK by the KRDS/I2S2 Digital Preservation Benefit Analysis Tools Project [34] funded by the Joint Information Systems Committee (JISC) that, combining partners from institutions, active research projects, and disciplinary data services, developed a toolkit for establishing a cost activity model and benefits framework in order to better define the value chain and benefit analysis of digital preservation, specifically centered on preservation of research data. The joint efforts of the Blue Ribbon Task Force and KRDS initiative helped with defining a reference model for digital preservation, a model which aimed to address the whole economic life cycle of digital assets and not only those actions related to archiving. Among other interesting ideas, all these economicframed initiatives concluded how difficult it was to achieve economic sustainability due to the difficulty in coordinating benefits, incentives to preserve, and responsibilities across the community of stakeholders involved in the preservation of a particular set of digital material. Additionally, referenced literature states that efficient preservation of digital content should be tight to supply and demand rules, as economists tell us that without the demand for access, there will be no interest in preservation.

Digital information has gained its foothold and, in a matter of decades, patterns of creation and use have changed rapidly. On the supply side, repository initiatives mentioned at the beginning of this chapter, centralized or decentralized, offer a quite robust and continuously-under-development preservation infrastructure to manage digital information. On the demand side, most of it revolves heavily around short-term reuse, and there is an absence of well-articulated demand for the long-term run. Demand is about what people wants to consume, and such a statement guides us to the concept of value. The value of digital assets is best understood as what digital materials are good for, and that is usually understood as the ways that the materials are used-to advance knowledge, entertain or bring pleasure, help solve problems, or inform public policy. Thus, the demand for a digital object is tight to its value and the latter is often diffuse, as the criteria for selection has not been formulated. Preservation services can be supplied by one institution, or distributed across many but, in any case, an entity, centralized or a distributed, willing to know which objects have preservation priority in order to guarantee their supply would not know what to collect, for whom, or for what purpose. Therefore, the first challenge to preservation arises when demand is diffuse or weakly articulated. Addressing the matter of demand is always the first step in developing sustainable preservation strategies. In line with giving a response to the DP problem based on demand and value, Ref. [35] claims that investment is only undertaken after reflection on business cases and an assessment of the value that the action would bring to the organization. In creating a business case, the majority of people focus primarily on cost, but there must be a counter-balancing focus on value, and there are no good DP business cases to support that.

The work presented in this article leverages an object-centered paradigm vision with a cost-value balance in mind. Generalist approaches may result in poor decisions on how preservation investment is applied, and they can have major implications on how much information can be preserved and how effectively. Thus, the results presented aim to demonstrate the feasibility of a differential self-sustained preservation paradigm where digital objects (DOs) are intelligently programmed with cost-aware sensing skills. In our approach, SPDOs manage a given budget to assure their survival in the long-term, by negotiation with storing repositories and web services by means of auction mechanisms. Once the initial budget is spent or close to, DO owners (private individuals, institutions, governments, etc.) may decide to refill specific budgets depending on the demand of the object itself, thus guaranteeing the survival of those ones valued, efficiently lowering overall preservation budgets. In an open data context or cases of digital material of public interest, open budget tracking may be available, thus encouraging users, content consumers and communities to make donations, increasing budgets to ensure preservation of valued content. The authors think that the presented bottom-up cost-aware budgetbased model would be able to cope better with the exponential growth of preservation needs. This article is a continuation of the works discussed in [36], focused on the budget 
optimization of SPDOs through micro-negotiations, and the work detailed in [15], centered in the development and testing of SPDOs through nature inspired computational intelligence methods. The presented article expands previous work on the field, searching for an efficient cost-management approach to be applied inside a self-preserving digital object context.

\section{The Self-Preserving Digital Objects (SPDOs) Paradigm}

Discussion carried out on previous chapters demonstrates that repositories, evolving from centralized to decentralized structures, together with the technology used to program them are necessary in order to guarantee digital preservation in the log term. The problem, which is the focus of this article, is to set up a sustainable strategy to preserve digital information over time, ensuring accessibility, readability, and execution independently of the software and hardware used to access it. An ever-increasing amount of the world's cultural and intellectual output is currently being created in digital formats, much of it publicly available on the Internet. Thus, digital architectures such as the Internet are ahead in this transformation. Opposite to top-down thinking, one of the most important features of the net is that it values the information, the object, above the containment structures used to store it, so information content is more important than the system used to store it. AI is playing a great role in this transformation process. Together with machine learning, they can be put to use in digital preservation across the business function in categorizing, driving actions instantaneously, or managing security settings. Taught by humans, AI will drive new efficiencies, allowing DOs, individual files or data repositories, to be programmed as intelligent self-sustained agents. The presented article explores a new paradigm in the field of DP, taking advantage of both decentralization and artificial intelligence, to provide the digital objects with the tools for self-preservation, a new context where digital objects share a part of the responsibility for preservation: given a set of rules, constraints, and finite economic resources, digital objects consume preservation services to guarantee their own survival inside the digital world, thus becoming SPDOs.

Nowadays, when a digital object is in need of preservation, there are two mechanisms available to grant it preservation: the preservation of the technological environment to assure archives' accessibility at any given time (format emulation, platform centered) and the file evolution to new formats to avoid obsolescence (format migration, object centered). The methods proposed in this article rely on the digital object itself to stay accessible, using preservation services to migrate and replicate into newer formats [37,38]. In the SPDO framework, the behavior of digital assets needs to be modeled in order to obtain the best preservation strategy. A design goal of the proposed paradigm is that, in the search for efficiency, digital objects become autonomous and active, separate from the server or repository where they are stored. SPDOs should be able to perform and respond to actions on their own and be active participants in their preservation. Making files intelligent opens the door for many future applications. In this sense, this article explores deeper inside the SPDO paradigm by programming digital objects with cost-aware preservation skills based on electronic auction behaviors. The proposed environment configuration allows files to contain all their code, data, and metadata. Related to self-sufficiency, the proposed preservation architecture makes digital objects fully mobile agents. That is, they can physically move from place to place, selecting the most budget-efficient preservation services in the network since they contain all the code, data, and support files they need. In order to demonstrate the feasibility of the proposal, the described environment has been simulated using a developed analysis software tool called Simulation Time Machine (TiM). Thus, for the experimental setup, cost-aware SPDOs, called CADOs, were created inside a network where numerous preservation services are available for purchase. Every CADO has a fixed budget of a given currency to spend on preservation services. Once the simulation begins, the timer starts and, from time to time, the simulator generates a software adoption wave (SAW). A SAW refers to either software or hardware shifts, and the consequences of a SAW usually entails the disappearance of a percentage of the DOs (obsolete), or a 
severe quality degradation of them, which hinders its accessibility. Immediately after a SAW, CADOs will use their budgets to request for preservation services, so they can maintain acceptable quality standards and staying accessible and readable by users. The features present in auction-based methodologies make them good candidates to be used for optimizing SPDO economic efficiency and open the door to future experimentation with other AI-based algorithms for preservation cost management. The article investigates the performance of auction-based mechanisms as an initial approach for developing cost-aware self-preserving behaviors for digital objects. In order to compare preservation strategies, CADOs are programmed with three different auctions mechanisms. The final objective of the experiments is to determine which mechanism offers the most efficient preservation strategy in the long run and, at the same time, demonstrate the feasibility of the proposed decentralized preservation paradigm.

\subsection{Software Adoption Wave (SAW)}

A wave simulates a massive update of old software for file management (editing, transformation, etc.) to new software, normally linked to technology (hardware or software) or market shifts. We can find examples of such changes periodically, like when Microsoft urges changes in common software like Office, .NET or Sharepoint. Today, most of the volume occupied by digital information is obsolete. The life expected from media is short, and there are many digital objects stored on dead websites. If we take a look at which digital products and services are the most used, a large portion of them did not exist five years ago. A particular format of a given medium can be expected to become obsolete within no more than five years [8]. Therefore, in the presented simulated environment, the period for a software update has been set to occur every five years, although longer or shorter periods could be proposed without loss of generality. Thus, for simulation-related purposes, this article fixed the SAWs occurrence at the same five-year pace.

Normally, it is common to find that a new version of software provides support for its previous software version, such support rarely exceeding more than three previous versions. Thus, after an update, older versions either lose their compatibility (too old) or retain it at a quality cost. Following this rule, for simulating purposes, the experiments carried out in this article consider that a digital object is likely to become obsolete after 15 years (3 SAWs, one every 5 years) if it does not receive any kind of preservation service. Before the 15-year critical mark, after each 5-year SAW, CADOs must hire preservation services to recover quality loss suffered during the wave. For the presented proposal, authors define the quality of a digital object as the arithmetic mean of four particular attributes associated to the CADO: accessibility, readability, integrity and authenticity. Initial attribute values of CADOs are fixed as a set up parameter at the beginning of the test. Once the simulation begins, after each SAW, random percentage amounts of value loss will be applied to randomly selected CADO's attributes, such loses ranging from $10 \%$ (least severe) to $20 \%$ (most severe) loss of attribute value, and thereby decreasing the overall quality of the CADO. The more quality a digital object loses, higher the risk of becoming obsolete. Given the numbers presented, in the best case scenario, after a 3 SAW period (15-year critical mark), the value of a particular CADO's attribute could lose a total of 30\% of its initial value. In the worst case scenario, after a $3 \mathrm{SAW}$ period (15 years), the value of a particular CADO's attribute could lose a total of $60 \%$ of its initial value. In order to force CADOs to actuate even in the best scenario, the proposed model considers CADOs to be in serious danger of obsolescence if, during the simulation, any of the mentioned value attributes falls below $70 \%$ of its initial value. Such a threshold means that, even in the best case scenario, no CADO will be left without access to preservation services for more than 15 years. The total time of TiM auction module experimental simulations has been set to 100 years (20 SAWs), time enough to analyze several SAWs and the performance reaction of the selected electronic auctions methods in terms of budget efficient management and quality assurance. 


\subsection{Cost-Aware Digital Object (CADO) Behavior}

As stated before, a CADO is a cost-aware digital object equipped with the necessary tools and programmed for self-preservation. A CADO represents the central element for understanding digital preservation from an object level perspective, as the object itself disposes from financial means for self-preservation in the form of a budget. The proposed TiM environment where simulated experiments will take place considers CADOs as different file types coexisting in various formats. CADOs belong to particular nodes of the network, users, or public/private storage sites. During the simulation, as time passes and SAWs happen, the quality of CADOs deteriorates. As stated before, the quality of a digital object is computed as the arithmetic mean of four particular attributes associated to the CADO: accessibility, readability, integrity and authenticity, the combination of them representing the quality of the file, tied to its potential digital longevity. At any one time, the digital object starts being at risk of becoming obsolete. As stated in the previous section, for simulation purposes, we consider CADOs to be in serious danger of obsolescence if, during the simulation, any of the quality-defining value attributes falls below a threshold value of $70 \%$ of its initial value. When this condition is met, CADOs start searching for available preservation services in order to restore damaged attribute values and restore the overall file quality. Depending on the auction method used to program the CADO behavior, different strategies for quality assurance and budget management will arise. The negotiation of digital preservation expenses will depend on the particular preservation needs of every CADO.

In purpose of standardization of the results obtained, easy operation and aiming to universalize the price assignment of the LTDP services costs, the budget assignment to each CADO is given in the form of a specially defined virtual token called PRESERVA (P). PRESERVA tokens do not exist nor they are listed in any virtual stock market and they are only used as an experimental digital currency for preservation research purposes. For expenses below $1 \mathrm{P}$, fractions of PRESERVA, called miliPRESERVA (mP), will be used, being $1000 \mathrm{mP}=1 \mathrm{P}$. For the simulated experiments presented in this article, tests will be repeated using three different initial budget amounts given to CADOs in order to test the capabilities of proposed auction mechanisms under variable initial budget circumstances.

\subsection{Preservation Services}

Nowadays, most well known digital repositories offer little support for actual preservation activities. As a consequence, a range of independent software tools have been developed for individual preservation tasks or services. Services is a term with different contexts of use and has lately become very popular through the service oriented architecture (SOA) concept. In our decentralized preservation context, preservation services break digital preservation into smaller manageable tasks, fulfilling specific preservation needs in an efficient way. This approach, called microservices [39], has become particularly relevant in distributed environments and also proven very useful for smaller institutions or projects that do not have the capacity to develop solutions covering all preservation functions. Specialized services can help to resolve a granular issue without compromising any of the primary functions of the preservation system, allowing flexibly into combining specialized solutions for preservation depending on the requirements of the client. Advantages of splitting preservation services into specialized agents are numerous. Since each of the services is small and self-contained, they are collectively easier to develop, deploy, maintain, and enhance. Equally as important, they are more easily replaced when they have outlived their usefulness. Although the individual services are narrowly scoped, the complex function needed for effective curation emerges from the strategic combination of individual services. Over the last ten years, different initiatives promoting microservices for digital preservation have appeared, both in the public and private scenes. Some public examples are the works at the California Digital Library [40], the Integrated Rule-Oriented Data System iRODS of the Electronic Records Archives at the US National Archives [41], 
the community driven Safety Deposit Box (SDB) [42] or the open archival information system Archivematica [43].

Previous sections defined the quality of a CADO as a combination of four individual attributes: accessibility, readability, integrity and authenticity, each value ranging from $0 \%$ to $100 \%$. A CADO with an accessibility attribute value of $100 \%$ means that this digital object is $100 \%$ reliable in terms of its accessibility. On the other side, an accessibility attribute value of $0 \%$ means that the file cannot be accessed in any way. For the simulated results presented, an attribute value loss below $70 \%$ of its initial value indicates that the CADO is in serious danger of becoming obsolete. If, after a given SAW, the CADO emerges with any of its individual attribute values falling below the $70 \%$ mark, it will immediately start searching for specific preservation services in order to restore the quality lost by hiring a service and fixing the damaged attribute. In order to model the preservation services, following the microservices approach, preservation services have been organized into families, grouping preservation services dedicated to recover each of the quality-defining attributes of the CADOs. Thus, the simulator generates specific services for enhancing the accessibility, the readability, the integrity or the authenticity of objects in need. Another feature of microservices is that each of them, depending on estimates of real market prices defining the service family they belong, offers a particular maximum price and a minimum one for their services at any given auction. With the aim of generating variability and opportunities among offered service prices inside a family, resultant maximum and minimum offers of each microservice are generated randomly using a normal probability distribution function $X \sim \mathcal{N}\left(\mu, \sigma^{2}\right)$, where the mean $\mu$ represents the real market estimate of the maximum and minimum prices and a variance $\sigma^{2}$ fixed to $\pm 10 \%$. The simulated microservices model also links every offered service with a random amount of the attribute's value restoring capability. The percentage value increase for a CADO attribute offered by a given microservice is also randomly generated using a normal probability distribution function, with a mean based on market estimates and a variance set to $\pm 10 \%$ [39].

\section{Auction-Based Methods}

Internet and its associated technologies have significantly changed the way people exchange information and participate in business transactions. Participating actors have enabled electronic commerce business models whose reach and scope were unattainable in traditional markets. Web-based online commercial activity for electronic auctions dates back to 1995, when two auction sites were founded independently with alternative business models, OnSale [44] and eBay [45]. Since then, the scope and reach of these auctions models have been propelled by the Internet to a level beyond what the initial purveyors had anticipated. eBay, the premier online auction retailer, reported $\$ 100.0$ billion in gross merchandise volume (GMV) in 2020 [46]. One of the main reasons for such success is because online auctions are favored by Internet decentralization, which removes the physical limitations of traditional auctions such as geography, presence, time, space, and a small target audience. An electronic auction (e-auction or online auction) is a transaction between sellers (the auctioneers) and buyers (the bidders) that takes place on an electronic marketplace and allows buyers to bid online for auctioned products or services according to a published specification. Depending on the actors involved, e-auctions are performed as business to business (B2B), business to consumer (B2C), or consumer to consumer $(\mathrm{C} 2 \mathrm{C})$. In the context of this article where an ecosystem of SPDOs and digital preservation services interact in a decentralized environment, CADOs will perform as bidders that, in a time of need, search for the best preservation service available (auctioneers). To do so, services belonging to each one of the previously described families of services offer their preservation products to CADOs by means of an auction system. As the simulated results point out, CADO budget efficiency is highly dependent on the auction strategy chosen. Like physical auctions, online auctions come in a variety of types like ascending English auctions, descending Dutch auctions, first-price sealed-bid, Vickrey auctions, and others. For the simulation results presented, the proposed auction methods use a basic 
descending Dutch method in combination with a multi-unit auction and a combinatorial auction. With the purpose of demonstrating the goodness of the two electronic auctions methods proposed, results obtained with both auction strategies are compared with a simple direct purchase strategy, called direct purchase baseline. The results obtained with the best simple baseline strategy will be used as a reference value for comparison with the multi-unit auction and the combinatorial auction methods.

\subsection{Direct Purchase Baseline (DPB)}

With the aim of obtaining reference baseline results, three basic purchase mechanisms have been programmed. Once tested, the results obtained with the best simple direct purchase behavior will be used as reference benchmark to better compare and analyze the results obtained with the multi-unit auction and the combinatorial auction methods. Thus, the three basic purchase mechanisms are:

- Best Ratio. After a SAW, any CADO in need of a particular preservation service (any of its quality-defining attributes falling below the $70 \%$ threshold), among all the possible offers inside the desired family of services, will select the service with the best ratio of resultant quality increase to price of the service. In other words, the CADO will always choose the service which gives the highest percentage increase of a damaged attribute at the lower cost possible.

- Best Quality. After a SAW, any CADO in need of a particular preservation service (any of its quality-defining attributes falling below the $70 \%$ threshold), among all the possible offers inside the desired family of services, will select the service with the best resultant quality increase of the damaged attribute. Thus, the CADO will always choose the service that gives the highest percentage increase of a damaged attribute no matter the cost.

- Random Choice. After a SAW, any CADO in need of a particular preservation service (any of its quality-defining attributes falling below the $70 \%$ threshold), among all the possible offers inside the desired family of services, will select a random available service from a given family list of services. For this basic purchase mechanism, the CADO neither considers the price nor the quality of the service, its only criteria is to cure itself as fast as possible when preservation needs appear and therefore chooses the first randomly consulted service.

For the DPB tests, CADOs can only hire one service at a time. If, after a given SAW, more than one attribute falls below the threshold, the CADO will first search for curing the worst injured attribute and, in a second, third or fourth direct purchases, attend the rest of the damaged attributes in an ascending order. Additionally, all direct purchase approaches share a primordial rule: a CADO accepts a service price offer if, after following one of the three specific mechanisms criteria, the proposed price can be covered by the CADO's current budget. If at any given time during the simulation a CADO budget amount is not enough to purchase the initial first selected service (according to best ratio, best quality or random choice strategy), the CADO will look for the immediate second service available from the offer list, and so on, till it finds one it can pay (a CADO can afford a price of a service because its remaining budget can cover the service price). If the budget of a CADO is over or the amount left does not allow it to buy any preservation service available, then the CADO will not be preserved and its damaged attribute or attributes will remain below the threshold, driving the CADO inevitably to obsolescence as future SAWs will eventually continue to deteriorate more and more attributes, tanking the overall quality of the file.

\subsection{Reverse Multi-Unit Descending Auction (RMDA)}

A multi-unit auction is a type of auction in which several homogeneous items are sold [47]. Multi-unit auctions help avoid the lumpy bid problem [48], which arises when bidders can only compete on the basis of one bid, and improve allocation efficiency by allowing bidders to buy multiple units through a bidding process. Multi-unit auctions are often used by governments or big private companies for the allocation of numerous 
resources including company stocks, electricity generation contracts or nature conservation contracts. The units can be sold each at the same price (a uniform price auction) or at different prices (a discriminatory price auction). The present case considers the first option, and applies a multi-unit auction as a uniform price auction. The particular, reverse, feature of the proposed auction is that, in our experiments, CADOs in need of a specific attribute curation group together offering themselves as auctioneers to the correspondent family of preservation services, these ones acting as bidders. As a result of grouping, CADOs expect to find better prices from preservation services, as the latter compete for packs of digital objects to preserve by offering their best price to win a bundle of given services at once. The higher the number of CADOs inside a group, the bigger the group discount offered by competing preservation services. For the RMDA design, bids from each service will descent between a particular maximum and minimum service price according to a normal distribution, as detailed in Section 3.3. The auction starts with services bidding for their maximum offer and, following a predefined descending pattern, the price will get lower and lower until the group of CADOs accepts the bid or service minimum price is met. In order to accept the bid, grouped CADOs agree on a predetermined acceptance price based on their average budget disposals. Here, the rule for accepting a pack price offer from a preservation service is: A group of CADOs will accept a service price offer for preservation if that price is equal or lower than the initially agreed predetermined acceptance price. Before this condition is met, several bidding iterations may happen, as bidding family preservation services do not have any kind of information regarding grouped CADOs budgets. Acting this way, grouped CADOs can get better prices compared to the ones they would get in an individual auction, where they, according to the defined model, would be forced to accept preservation service offers as soon as their budgets could cover the expenses.

The RMDA simulation is structured as follows. After a SAW, there will be 4 independent and randomly ordered consecutive auctions, one for each quality-defining attribute (accessibility, readability, integrity and authenticity). CADO group size has no limit, all CADOs in need of any particular preservation service will group together as a multi-unit pack for each of the four auctions. In the RDMA, the CADOs can only participate in one auction at a time. If, after a given SAW, a CADO has more than one attribute below the critical threshold (70\% or lower), it will first group with other CADOs participating in the auction offering preservation services for its most deteriorated attribute. Once the attribute has been preserved, the CADO will participate in the next needed auctions to try to preserve the rest of its damaged attributes in an ascending order. Each auction uses a descending offer Dutch mechanism [49], where services bid simultaneously, starting at their particular variable, normally distributed, maximum price. If two or more preservation services meet the winning bid at the same time, the CADO group will choose the service with the best ratio between the attribute value increase given by the service and the winning bid price. With the aim of modeling group advantages, the number of CADOs that are part of a given group directly affects the final price of the preservation service. Therefore, for every CADO inside an allotted pack after the first one, the price of the service will receive a $1 \%$ discount, up to a maximum of $10 \%$, with respect to the winning bid price. As an example, a group of 5 CADOs would get a $4 \%$ discount whereas a group of 15 CADOs would get a $10 \%$ discount. After the first 4-auction-round, if there are still CADOs in need of preservation as well as services which have not reached their minimum service price yet, another 4-auction-round will take place. Remaining CADOs (from the same attribute auction and others coming from previous attribute auctions) will pack again and bids will resume, and so on, until either one of the following scenarios is met: (1) There are no more CADOs with preservation needs or (2) the preservation services have all reached their minimum service price and might not cut their prices any further. The second scenario leaves us with CADOs whose budget is over or with amounts left lower that the cheapest service price offered. Additionally, in the second scenario, there also exists the possibility of one single remaining CADO with still some budget left and not other CADOs to group. 
In that case, a simple ordinary descending auction with no discounts will take place until either a price is meet or minimum service price across all available services is reached. The remaining CADOs will not be preserved, and their damaged attribute or attributes will remain below the threshold, driving the CADOs inevitably to obsolescence as future SAWs will eventually continue to deteriorate their quality.

\subsection{Combinatorial Descending Auction (CDA)}

A combinatorial auction or multi-lot auction is a type of auction in which participants can place bids on combinations of discrete heterogeneous items, also called packages or lots, rather than individual items. Combinatorial auctions have been used for many years in estate auctions, where a common procedure is to accept bids for packages of items. Recently, they have been used for industrial procurement, allocation of radio spectrum for wireless communications, truckload transportation or bus routes [50]. The study of combinatorial auctions is inherently interdisciplinary, lying at the intersection of economics, operations research, and computer science. One of the interesting ideas behind combinatorial auction lies in the fact that packages of products may be more attractive to bidders as the valuations on bundles of items can be higher than the sum of the valuations of individual items of the combined package. In the proposed CDA auction, CADOs in need of curation (acting as bidders) will only search for preservation services (auctioneers) if, after a given SAW, they have two or more under-threshold quality-defining attributes. This way, CADOs expect to find a better price opportunity in an auction where services are offered as variable-size bundles or service packages belonging to the four family of services (accessibility, readability, integrity and authenticity service families). Regarding valuation, preservation tasks often share specific properties and needs across families. It makes sense to admit that updates or improvements in the integrity of a file may result, a part from its correspondent integrity attribute upgrade, in a collateral upgrade in, for example, the readability of that file, not with the intensity a specific readability family service would do, but it would definitely increase the value of the attribute at a certain degree. With this assumption in mind, the proposed CDA simulation gives a fixed collateral valuation boost (more attribute value increase) for CADOs hiring a multiple family service bundle. For the CADO acquiring the package, this boost results in a higher quality upgrade than the one it would get if hiring stand alone services one by one. For the CDA design, after each SAW, the simulator will offer all service package combinations (two, three or four service packs) to demanding CADOs. Bundle maximum and minimum prices are computed as the summation price of individual maximum and minimum prices of all services included in the package, each value computed according to a normal distribution, as detailed in Section 3.3. The auction starts with bundled services at their maximum combined offer and, from there, they follow a predefined descending pattern. CADOs, as individual bidders, will accept bundles of services based on their ratio value between the resultant total quality increase (boosted) and the current offered price of the pack. Thus, for a given price, CADOs expect to get better packed service upgrades compared to the ones they would get in an individual, single service, auction.

The CDA simulation is structured as follows. After a SAW, CADOs with at least two under-threshold (70\% or lower) quality-defining attributes will individually start looking for bundles of grouped preservation services. The simulator will calculate all possible packed service combinations available for each demanding CADO and will list ordered bundle offers based on the ratio between its aggregated attribute value upgrade and its aggregated price, in an ascending order. Bundled attribute value upgrades are increased with respect to its default value by a percentage depending on the amount of services contained inside the package: $2 \%$ value increase for each attribute value in the case of a bundle pack containing two different services, 3\% value increase for each attribute value in the case of a bundle pack containing three different services and $4 \%$ value increase for each attribute value in the case of a bundle pack containing services from all four families. Listed ratios are calculated with boost additions. CDA simulated auctions use a 
descending offer Dutch mechanism and take place simultaneously, with packages starting at a variable, normally distributed, maximum price. Several auction iterations will take place until either one of the following scenarios is met: (1) There are no more CADOs with preservation needs, or (2) the preservation services bundle offers have all reached its minimum service price and might not cut their prices any further. The second scenario leaves us with CADOs whose budget is lower that the cheapest packed service price offered. In the case of a particular CADO still having some budget left enough to, at least, buy a single preservation service, a simple ordinary descending auction will take place with all available services listed by ratio in an ascending order (value attributes used to compute ratios are not boosted in single auctions). The most deteriorated attribute value will have priority for preservation. Finally, second scenario also comprises CADOs whose budget is over or with amounts left lower than any single minimum price offered by any existing preservation service. In such cases, CADOs will not be preserved and their damaged attribute or attributes will remain below the threshold, driving the CADOs inevitably to obsolescence as future SAWs will eventually continue to deteriorate their quality.

\section{Auction Simulator for DP Studies}

The Simulation Time Machine (TiM) [51] is a software tool developed at the TECNIO Centre EASY research group of the University of Girona for simulation of multi-agent systems (MAS) behaviors. Initially conceived as a simulated environment for running a wide number of agent-based ecosystems, today it has evolved to a simulation platform for DP studies in SPDO contexts. Recently, the TiM simulator has been used for testing DO behaviors using nature-inspired computation intelligence methods in a SPDO environment [15]. The platform, programmed in JAVA, has been developed as a modular platform. For the experiments presented in this article, the TiM platform has been extended to incorporate a new module: the auction simulator. The auction simulator allows users to recreate a simulated digital preservation ecosystem where CADOs interact with preservation services by means of user-defined auction models. The new auction module has been structured into two layers:

- $\quad$ Core layer. It contains the main classes that run the auction processes and other libraries for data management and export, comparators and search optimization engines.

- Interface layer. This layer contains all the tools and menu options for user interaction: choose auction type, launch simulation, view results and export. This layer uses Primefaces technology for data validation at client side.

The application workflow of the auctions module inside the TiM simulator is structured around three main functionalities: first, the input synchronization function is in charge of importing the initialization parameters and all needed information to launch auctions, number of CADOs to be preserved, auction strategy, the preservation services available or the features describing the SAW periods. This functionality also adds statistical variability to the system according to a list of distribution function model generators, selected as initialization parameters. Second, the launch auction function deals with the execution of the simulation once all parameters have been correctly set. Finally, the results of the simulation are saved in a file. The export information function processes all the gathered data during the simulation to make it available to export into different output formats.

The TiM software is currently in development. Once finished, it will be open and free to use by the community. The TiM simulator has been used as a software platform to simulate the interactions of CADOs with preservation services in a distributed environment. The simulator allows users to analyze the effectiveness of multiple preservation strategies. In this article, the TiM simulator has been used to test and compare simple direct purchase strategies with auction-based mechanisms, as described in Section 4: three simple direct purchase baseline strategies (DPB), a reverse multi-unit descending auction (RMDA) and a combinatorial descending auction (CDA). 


\subsection{Simulation Set $U p$}

As detailed in Section 3.1, the TiM total simulation time has been set to a 100-year period, with SAWs taking place every 5 years, for a total number of 20 SAWs in each simulation. With the aim of simulating real quality starting conditions, in all the experiments, CADOs start with a total quality value of $75 \%$, with all attributes having exactly a value of 75/100. This initialization value setup assures that most of the CADOs will have one or more attributes below the critical threshold $(70 \%)$ at the first SAW iteration, forcing them to start looking for preservation services immediately. After every SAW, the resultant quality of each CADO is evaluated. As explained in Section 3.2, the total quality of a digital object is computed as the arithmetic mean of four particular attributes associated to the CADO: accessibility, readability, integrity and authenticity. SAWs randomly deteriorate attribute values, damage ranging from $10 \%$ (least severe) to $20 \%$ (most severe) loss of attribute value. As described in Section 3.3, available preservation services are grouped in four families, each one offering specific preservation services tied to one of the four quality-defining CADO attributes. Each preservation service max-min price and attribute value upgrade is generated randomly using a normal probability distribution function based on market estimates and a variance set to $\pm 10 \%$. After each SAW, CADOs with one or more attribute values below the critical value loss threshold (70\%) automatically start looking for preservation services according to each defined scenario. The environment conditions have been defined as equal for all simulated scenarios: 1000 CADOs and 100 preservation services ( 25 of each family) interacting along a 100-year period simulation time. All the simulated scenarios detailed in the next lines have been repeated a total of five times, with the results shown for every scenario being the resultant mean of these five repetitions.

The simulated demonstration presented has been divided into two phases. The first phase compares simulation results obtained with three basic purchase mechanisms, called DPBs mechanisms. The results obtained with the best simple direct purchase behavior will be used, in the second phase, as reference benchmark to better analyze the results given by the multi-unit auction, RMDA, and the combinatorial auction methods, CDA, all tested under the same environmental conditions and constrains. Additionally, in both phases, three different budget scenarios are considered: low, medium and high budget scenario. Low budget scenario represents a particular situation where CADOs are given a tight budget for their self preservation, according to current market price estimates (the estimated lowest amount needed to pay for preservation services during 100 years). The medium budget scenario represents a particular situation where CADOs are given an average budget for their self preservation, according to current market price estimates (an average between estimated lowest and highest amount needed to pay for preservation services during 100 years). Finally, high budget scenario represents a particular situation where CADOs are given the highest budget for their self preservation, as it corresponds to the quantities needed by CADOs to be able to pay for preservation years during 100 years, paying for each service a maximum estimated cost. Following the monetary standardization proposed in Section 3.2, $500 \mathrm{mP}$ (low), $1000 \mathrm{mP}$ (medium) and $2000 \mathrm{mP}$ (high) have been assigned to each CADO in the three presented scenarios.

Table 1 shows the initial parameter configuration to run on TiM for every scenario. The Common to All parameter section applies for the two phases of the simulation: the first phase where three basic purchase mechanisms are compared, and the second phase, where the best DPB is used as reference benchmark to compare the two auction negotiation systems, the RMDA and the CDA. The second half of Table 1 is about the particular parameters used during the second phase of the simulation, concerning design features for the RMDA and the CDA mechanisms. These parameters were introduced as the initial setup conditions for each of the purchase mechanisms tested during the simulated experiments detailed in the results section of the article. The final selected values presented in the table above were tuned experimentally. All experimental tests were performed on a laptop with the following configuration: Intel(R) Core(TM) i7-4500U CPU @ $1.80 \mathrm{GHz}$ and 8 GB of RAM memory. The simulations always took under $45 \mathrm{~s}$ to execute. Timing was not a constraint for 
the development of the article. In the future, if the complexity of the algorithms increases, better hardware settings would improve the simulation times.

Table 1. Definition of the initial parameters used in the TiM simulations for every scenario.

\begin{tabular}{|c|c|c|}
\hline Parameters & & Common to All \\
\hline Total simulation time & & 100 years \\
\hline Equivalence of 1 simulation step & & 1 SAW (5 years) \\
\hline Total number of SAWs & & 20 \\
\hline Total number of CADOs & & 1000 \\
\hline Total number of preservation services & & 100 \\
\hline Random attribute value damage range & & $10 \%$ to $20 \%$ \\
\hline Attribute value upgrade percentage & & $X \% \sim \mathcal{N}\left(\mu, \sigma^{2}\right)$ \\
\hline Starting CADO total quality value & & $75 \%$ \\
\hline Attribute value loss critical threshold & & $70 \%$ \\
\hline \multirow[t]{2}{*}{ Budget scenarios (low, mid, high) } & & $500 \mathrm{mP}, 1000 \mathrm{mP}, 2000 \mathrm{mP}$ \\
\hline & RMDA & CDA \\
\hline$\%$ group discount & $1 \%$ to $10 \%$ & \\
\hline $\begin{array}{l}\text { Minimum number of under threshold } \\
\text { attributes to participate in auction }\end{array}$ & & 2 \\
\hline$\%$ Collateral valuation boost & & $2 \%, 3 \%, 4 \%$ \\
\hline
\end{tabular}

\section{Results}

In the presented experimental study, we were interested in: (1) understanding the results obtained from each auction-based method in comparison with direct purchase mechanisms; (2) finding which purchase mechanism provides the best average preservation capabilities in the long run (higher average CADO total quality over the 100-year simulated period); (3) finding which mechanisms show better average budget management capabilities (higher average remaining budget over the 100-year simulated period); and finally, (4) determine which of the proposed mechanisms offer the best combination of the two measured indicators, overall quality and budget management. In order to see how the behaviors based on the different purchase proposals worked as solutions for DP, the results are presented as a concise numerical study based on the following items, which we considered key to characterize the obtained results:

- The presented experiments analyzed how the models responded to a fixed 100-year simulation. As explained in Section 3.1, the period for a software adoption wave has been set to occur every 5 years, thus resulting in a complete simulation containing a total of 20 simulation steps, one after each SAW. At every simulation step, all needed purchase interactions based on selected behaviors are performed. At the end of every step, resultant total quality of CADOs and their respective budgets is extracted for mean value calculation. Finally, each simulation has been repeated 5 times and the graphical results shown depict average values (quality and budget) of these repetitions.

- Three different budget scenarios are considered: low, medium and high budget scenarios, and all the purchase mechanisms tested have been submitted to these budget scenarios. The final numerical results regarding quality and budget management extracted from each experiment are used for performance comparison.

Sections 6.1 and 6.2 show the results obtained in the experimental tests according to environmental conditions and constrains detailed in Section 5.1. First, the results on the DPBs mechanisms are presented. Next, the best DPB method is used as reference baseline for comparison with the multi-unit auction, RMDA, and the combinatorial auction method, CDA. 


\subsection{Phase 1: Results on the Direct Purchase Baseline (DPB)}

In the DPB simulations, five independent executions were carried out for each of the three budget scenarios, low, mid and high. Results depicted are mean values of these five executions. For this simulation, the three proposed direct purchase strategies are compared: Best Ratio, Best Quality and Random Choice. Figure 1 shows the simulation results obtained for the DPB mechanisms under a low budget scenario.
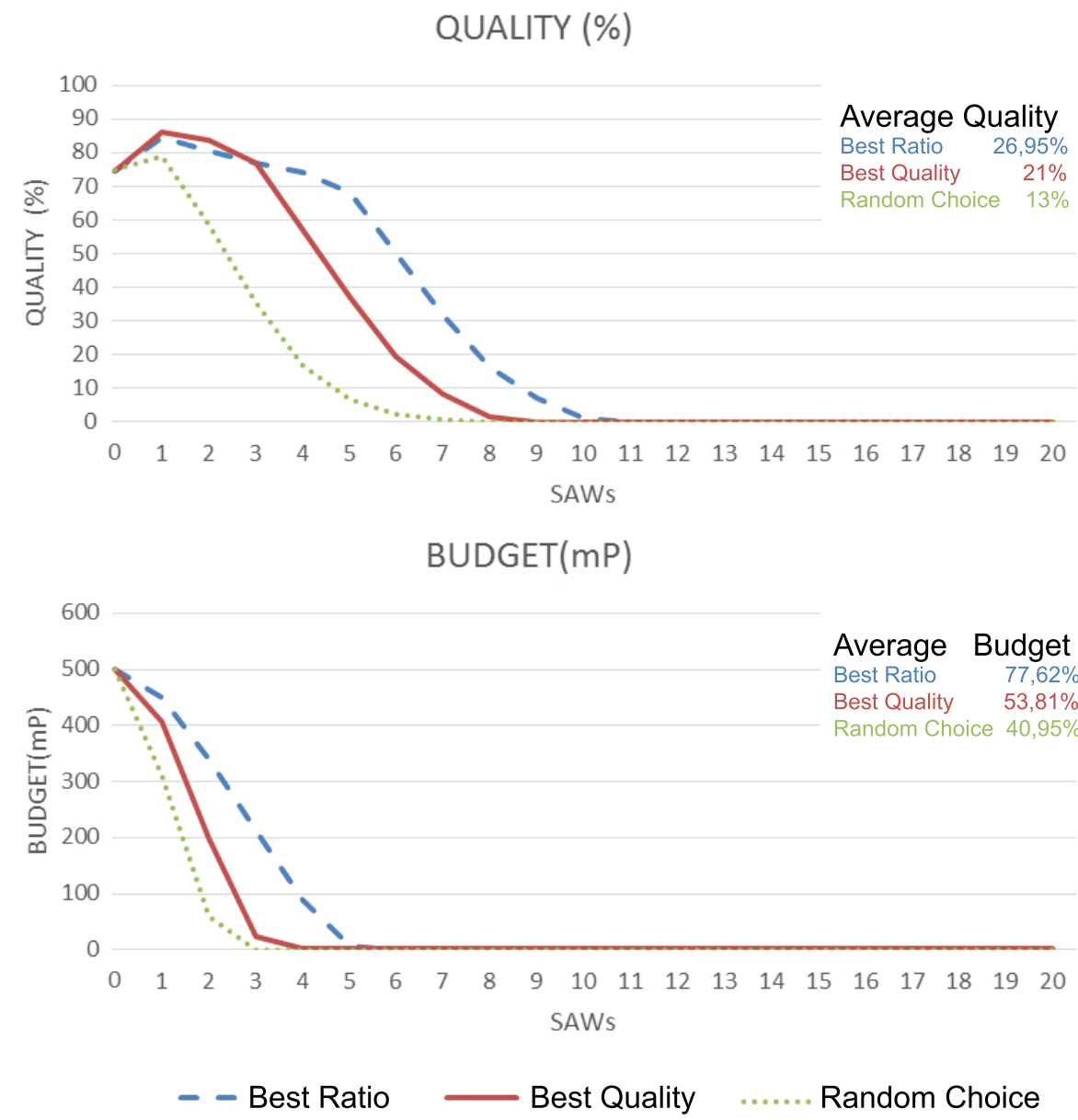

Figure 1. DPB results under low budget conditions.

As depicted in Figure 1, the plot illustrates the average resulting total quality and budget of all CADOs along 20 SAWs time, which corresponds to a 100-year time period. These results have been obtained under an initial low budget constrain of $500 \mathrm{mP}$. If we take a closer look to the results, the direct purchase strategy based on best ratio, higher quality increase to price of the service ratio, is the one obtaining the best results in both plots, either in terms of overall quality and budget lasting. Random choice strategy appears to be the one with the worst results while best quality strategy shows a behavior in between the best ratio and the random choice options.

Figure 2 shows the results obtained in the case of an initial medium budget constrain of $1000 \mathrm{mP}$. As can be appreciated, from the point of view of the collective performance, the results are similar to the ones obtained in Figure 1 with a low budget constrain. The overall quality pattern repeats, with the best quality strategy performing slightly better than best ratio while budget lasts, but falling abruptly once there is no more budget to spend. The random choice strategy continues to show inconsistency, rapidly spending all available budget with poor results. 

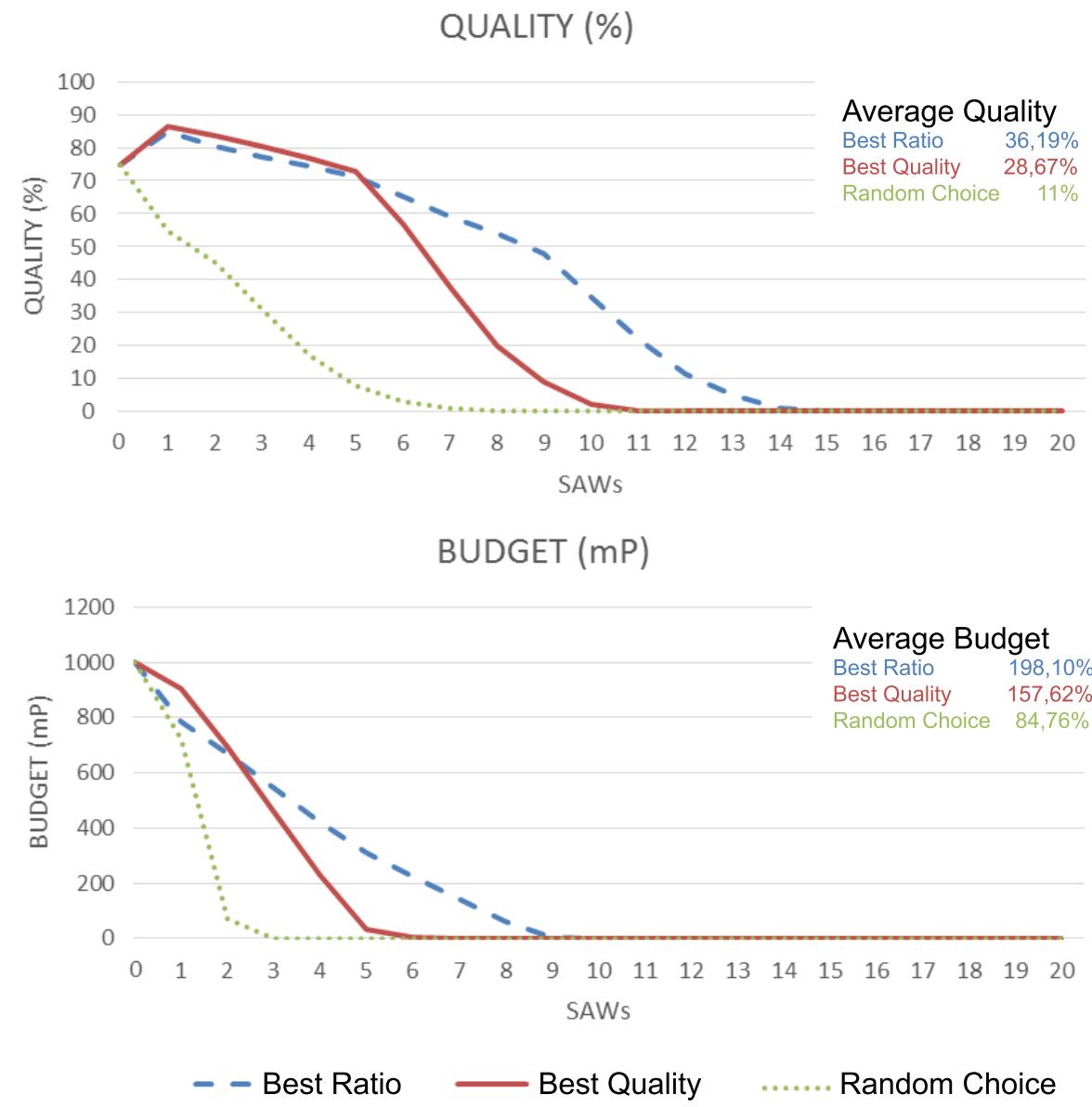

Figure 2. DPB results under medium budget conditions.

Finally, to end with the experiments related to phase 1, Figure 3 shows the results obtained in the case of an initial high budget constrain of $2000 \mathrm{mP}$. Here again the tendency repeats, with the best quality strategy performing slightly better than best ratio while budget lasts, but falling abruptly once there is no more budget to spend. This time, best ratio strategy manages to extend budget at a greater distance from best quality performance, maintaining acceptable quality levels until SAW number 16. Random choice strategy does not manage to get past 3rd SAW even in an abundant budget context.

The experimental results obtained during phase 1 point out best ratio strategy as the best direct purchase strategy among the three studied approaches in all scenarios. Although the overall CADO quality levels is slightly lower than those shown by the best quality strategy, the budget management capabilities demonstrated by the best ratio strategy significantly outperforms its rivals, allowing CADOs for extended time frames where they can be considered digitally preserved. In the high budget scenario, CADOs under best ratio strategy get to the 100-year mark with still some amount of quality left. For these reasons, the best ratio direct purchase strategy has been chosen as the direct purchase baseline strategy to be compared with the two proposed auction-based mechanisms during the second phase of the simulated experiments. 

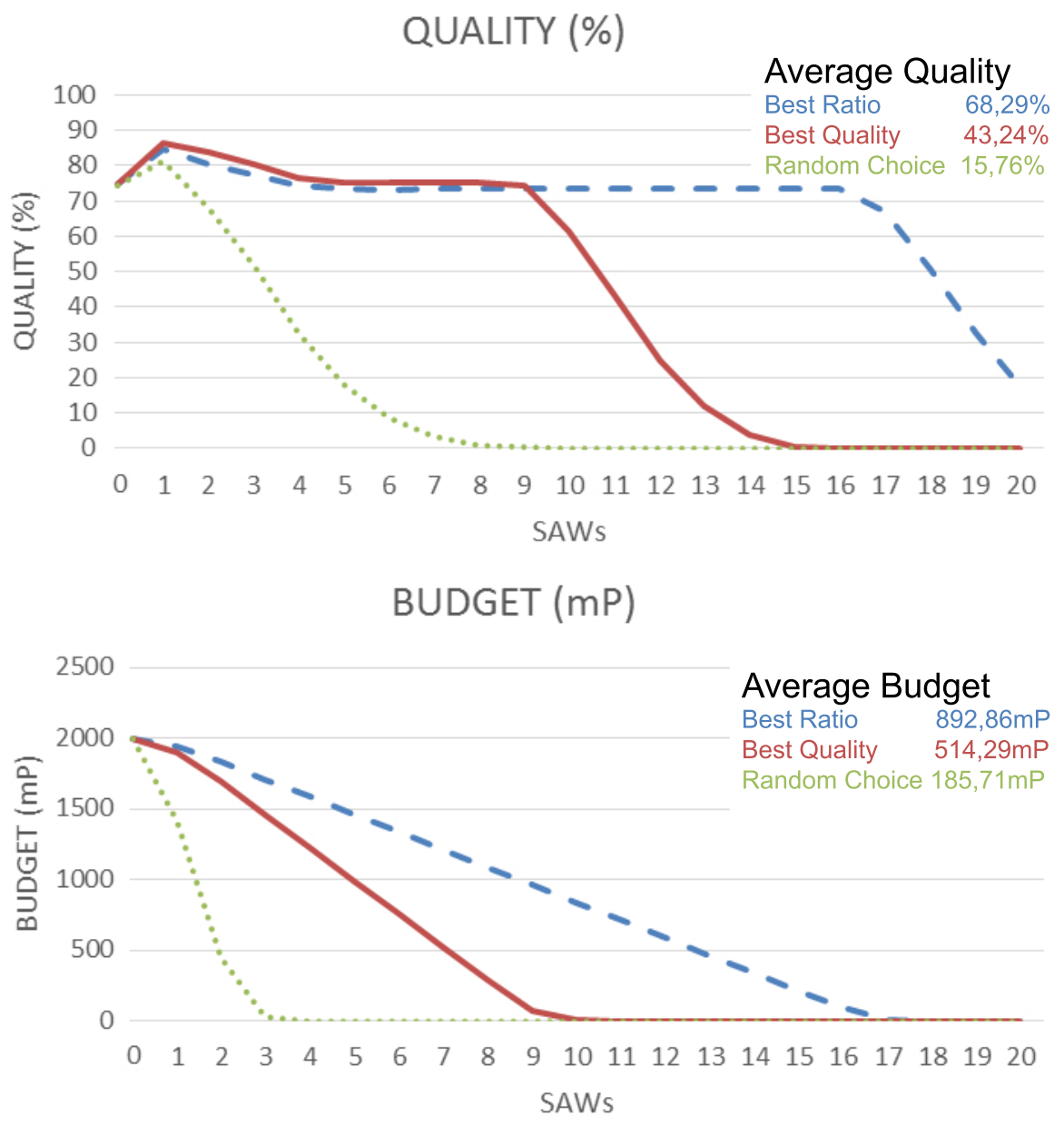

- - Best Ratio — Best Quality

Random Choice

Figure 3. DPB results under high budget conditions.

\subsection{Phase 2: Results on the Auction-Based Mechanisms}

For the auction-based simulations, five independent executions were carried out for each of the three budget scenarios, low, mid and high. In each execution, the multi-unit auction RMDA and the combinatorial auction methods CDA are compared together with the best ratio direct purchase strategy, acting as reference baseline, which demonstrated top performance according to results obtained in Section 6.1. Results depicted are mean values of these five executions. The simulated results obtained shown in Figure 4 compare the behavior of these three methodologies under a low budget constrain scenario.

The plot illustrates comparative results of total quality and budget of all CADOs along 20 SAWs time, which corresponds to a 100 -year time period. These results have been obtained under an initial low budget constrain of $500 \mathrm{mP}$. As depicted in Figure 4, for the first comparison, the CDA mechanism is the one obtaining the best results in both plots, either in terms of overall quality or budget management. The best ratio direct purchase strategy, acting as reference baseline, shows an average behavior compared to the two auction-based methods, with performance results lying between them. The RMDA mechanism appears to be the worst of the three, holding quality standings during the initial SAWs, but rapidly spending available budget, leaving CADOs with poor economical capabilities for accessing the preservation service market and, as a consequence, unable to restore their quality after the third SAW. Another important detail to discuss about these results is the remaining budget left on CDA, which starts at SAW 11 and continues onward. As it can be appreciated, this remnant is not used for hiring any preservation service despite all CADOs having overall quality values at zero or close to it, meaning that, 
at this point, there is a large portion of CADOs with some budget left but not enough to buy any preservation service, as the remnants are lower than offered minimum prices. On the contrary, RMDA behavior, although performing worse at budget management and quality returns, spends all available resources for most CADOs.

\section{QUALITY (\%)}

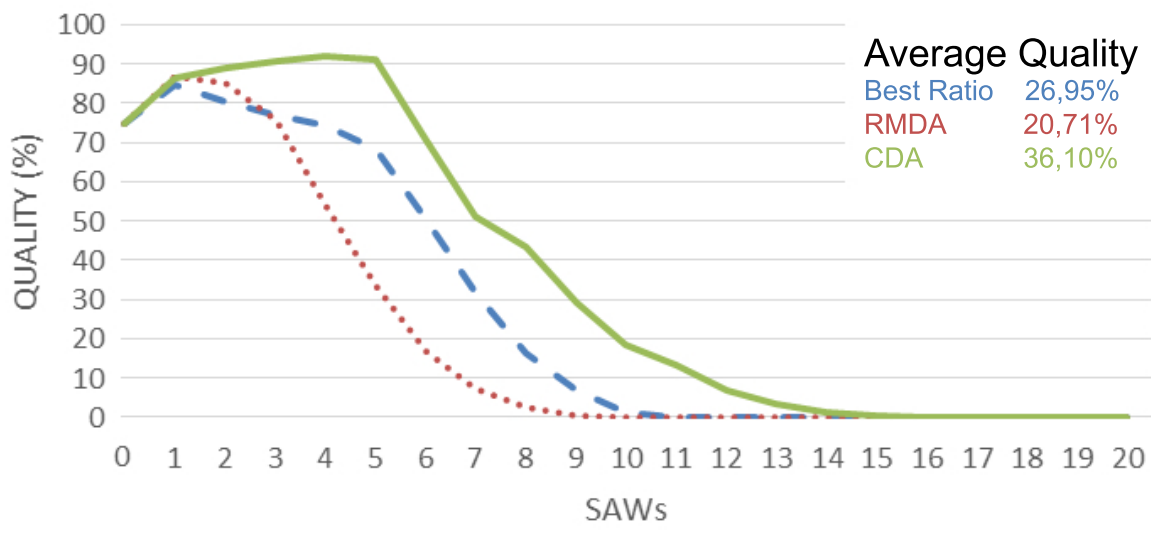

\section{BUDGET (mP)}

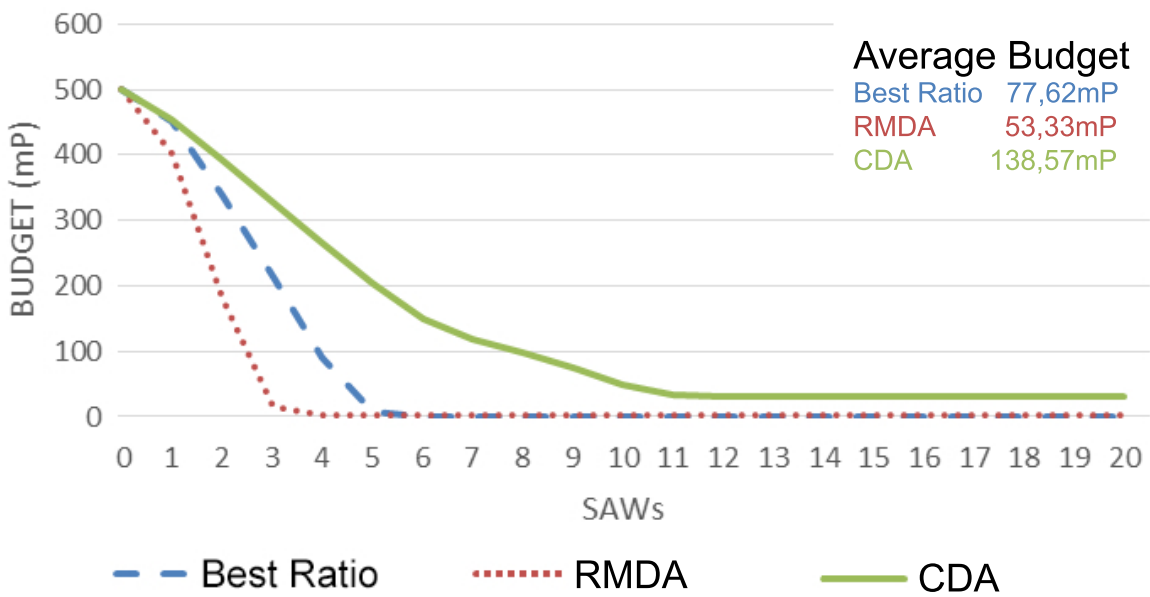

Figure 4. Auction-based comparison results under low budget conditions.

Figure 5 shows the comparative results obtained in the case of an initial medium budget constrain of $1000 \mathrm{mP}$. Again, from the point of view of the collective performance, the results confirm the tendency showed in Figure 4 with a low budget constrain. Here, the CDA mechanism continues to display the best behavior and, for the first time in all tests, CADOs get to the end of the total simulation time with acceptable quality levels, finishing at mean values around $40 \%$. The RMDA system continues to perform worse than the simple direct purchase mechanism either in terms of overall sustained quality and budget management. 

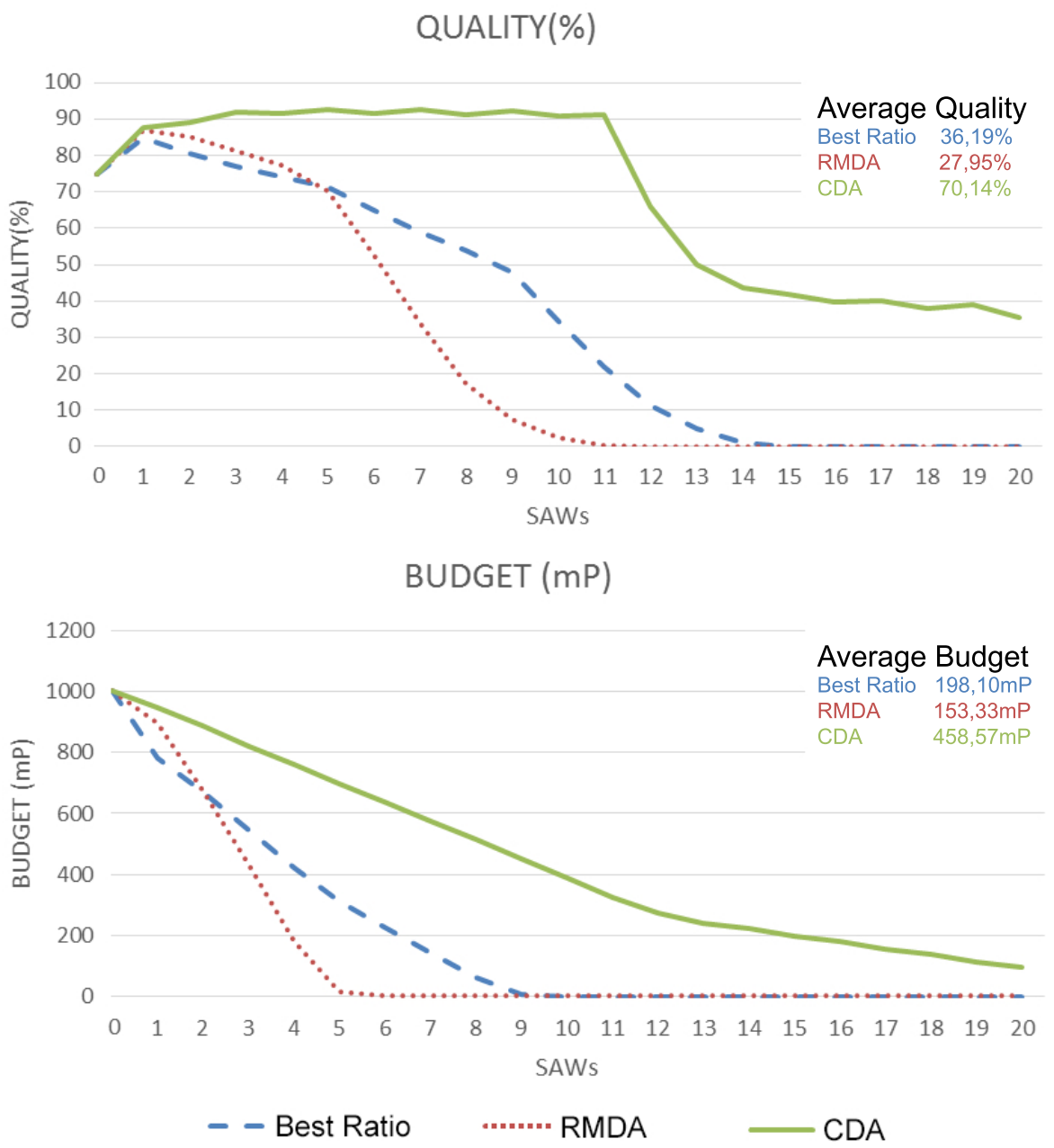

Figure 5. Auction-based comparison results under medium budget conditions.

Finally, to end with the experiments, Figure 6 shows the results obtained in the comparison case of an initial high budget constrain of $2000 \mathrm{mP}$. The results of this last plot confirm the previously observed pattern. The CDA behavior consolidates its position as the best of the three studied behaviors and now, when enough resources are available, is able to maintain total overall quality levels at good standards, with values circa $90 \%$. Results obtained by RMDA are again worse than the DPB method in terms of overall performance, but also in terms of consistency, denoted by the noticeable heavy saw pattern behavior in quality levels of Figure 6.

The analysis of the comparative results demonstrated that, in the context of low, medium and high budget disposals, on average, the best purchase mechanism in terms of sustained CADO quality and efficient budget management corresponds to the combinatorial descending auction (CDA) mechanism. This methodology is the only one showing acceptable quality values, for medium and high budget tests, at the end of the 100-year simulation period. Taking a look at the results obtained with the RMDA, the defined setup does not fulfill expectations as this auction method does not improve simple direct purchase behaviors inside the proposed paradigm. None of the proposed methods managed to make CADOs survive the simulations with initial low budget conditions. 

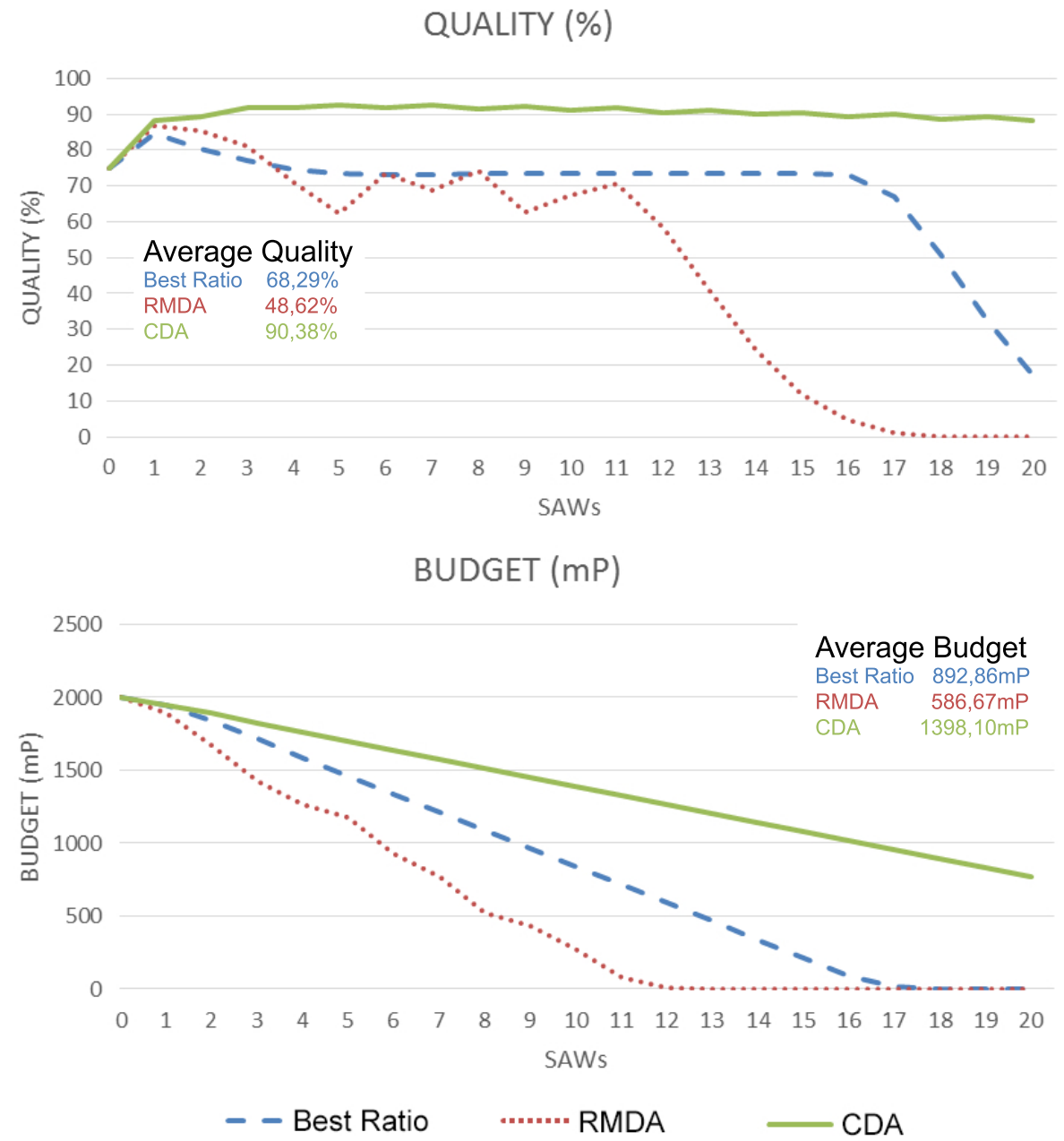

Figure 6. Auction-based comparison results under high budget conditions.

\section{Discussion}

The simulated results presented in this article aim to provide a graphical comparison as a measure of goodness of a new auction-based cost-aware sense for programming intelligent self-sustained digital objects inside the SPDO paradigm for the solution of the digital preservation problem. The proposed approach describes an environment where intelligent digital objects are programmed with cost-aware capabilities and budget management. Thus, SPDOs manage specific budget resources available in a distributed network to guarantee their survival in the long-term, negotiating with storing repositories and web services in order to find the best cost-efficient self-preservation strategy. Section 6 showed the performance of auction-based mechanisms and direct purchase strategies as an initial approach for developing cost-aware self-preserving behaviors for digital objects using the TiM simulator in three different budget scenarios: low, medium and high budget disposal. Furthermore, the simulated results obtained analyze the response of the auction models against a simple direct purchase strategy in every scenario. The evaluation of each budget management mechanism tested in this article gave the following conclusions:

- $\quad$ Direct Purchase Strategies (DPB): The experimental results obtained during phase 1 proposed the best ratio, which chooses the service offering the criteria as the best direct purchase strategy among the three studied approaches to be compared with the auction-based methods during the second phase of the simulated experiments. This purchase system is not an object of study in the article and is only meant to act as a baseline reference for comparing a common used purchase strategy against specific auction methodologies. 
- $\quad$ Reverse Multi-unit Descending Auction (RMDA): The simulated results obtained with the RMDA method did not offer any significant improvement compared to the selected DPB strategy. The proposed CADOs pack strategy, designed to help CADOs obtaining better service prices derived from grouping, systematically fell even below the DPB model in all tests. Future tests with multi-unit auctions will dig deeper into its formulation in order to propose alternative price acceptance policies and compare the results obtained.

- $\quad$ Combinatorial Descending Auction (CDA): The performance demonstrated by the CDA model is the best among the tested methods for all proposed budget scenarios. The foundations of this model allow for better individual budget management by the CADOs and, at the same time, acquire improved attribute quality upgrades by purchasing bundled services. As with the RMDA, the formulation used for the proposed model is very simple and authors believe that further research among variations will optimize current results towards the full potential of the method.

Qualitatively, comparing the results obtained with the three models tested under variable budget conditions of low, medium and high budget disposals, the best performance, on average, corresponded to the CDA method. This auction model was the only one that maintained high and even growing quality values during most part of the simulations and, at the same time, drew a gentle slope while managing available budget during the simulations. The results also point out that such a method suffers from budget allocation when particularly low levels are reached, as individual CADOs with low budget remains are quickly kicked by market dynamics because available services do not find any incentive in an individual operation below minimum price offers. In the case of the RMDA model, the analysis of the results revealed that a key parameter for this kind of auctions is the group's price agreement for bid acceptance. For these experiments, defined policy for group service price acceptance is based on average remaining budget on grouped CADOs. Thus, this average rises when higher budgets are available, draining resources quicker than it would do in a low average group. Such criteria punish wealthy CADOs instead of rewarding them for better budget management. On the other hand, the RMDA method has better results than the CDA when hiring services for CADOs with low budget disposal. Grouping capabilities make CADOs attractive for services despite poor individual price as the total price for the whole group can cover the service expenses and still generate some benefit.

\section{Conclusions and Future Work}

The objective of this research document is to present new results obtained as a continuation of the work published in [15]. That previous publication contained a proposal for a novel object-centered paradigm to address the digital preservation problem where digital objects, programmed with using basic computational intelligence behaviors, shared part of the responsibility for self-preservation. Thus, digital objects were programmed with the mission of their own long-term self-preservation, which means being accessible and reproducible by users at any time in the future regardless of frequent technological changes due to software and hardware upgrades (SAWs). Previous published results showed a comparison of different nature-inspired simulated behaviors interacting within a simulated environment using a specifically designed software platform called Simulation Time Machine (TiM). Digital objects are mainly files and, if they are programmed with the mission to persist and remain accessible, their strategy to do so is achieved by requesting preservation services spread among the Internet in order to guarantee the quality of their main defining attributes-accessibility, readability, integrity and authenticity-over time. In [15], the final simulated results obtained demonstrated that a digital preservation environment with objects programmed for self-preservation is possible. The experiments presented there were a first step towards creating real structured environments where models coexist under a predefined system of rules and constraints and concluded that more tests were needed in order to characterize the desirable properties that could be required 
from intelligent behaviors, the distributed interaction environment and the response of the preservation services. The results presented in the current work follow the opened path and add a new constrain to DO behavior inside the DP paradigm initially proposed. Now, migrations, copies and updates are not free to use by DOs, but are subject to budget limitations to ensure the economic sustainability of the whole preservation system, forcing the now renamed cost-aware digital objects or CADOs for efficient management of available budget in a distributed network to guarantee their survival in the long-term. CADOs will now negotiate with storing repositories and web services to find the best cost-efficient self-preservation strategy, thus becoming SPDOs.

New presented simulated results analyze DO performance facing finite economic resources. To deal with this added constrain, the presented approach compares auctionbased mechanisms and direct purchase strategies as intelligent behaviors attached to DOs. The proposed auction methods use a basic descending Dutch method in combination with a multi-unit auction (RDMA) and a combinatorial auction (CDA). With the purpose of demonstrating the goodness of the two electronic auctions methods proposed, results obtained with both auction strategies are compared with a simple best ratio direct purchase strategy (DPB). An improved version of the TiM simulator has been used to design the simulation environment for three different budget scenarios: low, medium and high budget disposal. The selected purchase strategies were studied taking into account two basic indicators: how well they performed in terms of total quality sustain and how well they behaved in terms of budget management. The direct purchase method based on best ratio strategy is used as reference baseline for comparison. The results show that the best performance, on average, corresponded to the CDA. This auction mechanism has obtained the best results in both indicators: best sustained CADO quality and better resource management in all three budget scenarios. It is important to note the poor performance showed by the RDMA method, even falling behind a simple direct purchase strategy. Certainly, the multi-unit auction design applied is very simple, and results would probably improve with more elaborated definitions, but the CDA implementation is also very simple and it greatly improved the results of all proposed simple purchase strategies. The results presented here are not meant to be a research work on electronic auction-based systems, but to take profit from this field as a tool for the study of strategies and behaviors for the self-preservation of digital objects. The results point out auction-based autonomous purchase systems as a good initial approach and open the door to test other efficient outlay behaviors.

Future work will look ahead to better exploit the features of the selected auction systems with the aim of improving its effectiveness. Future modifications include variations in the initialization setup of the selected auction methods, such as implementing better strategies for group's price agreement for bid acceptance in the case of multi-unit auctions or implementing negotiation models into CADO behavior in order to determine if a service price is too expensive, fair or a good deal according to current market prices. Additionally, from the preservation services point of view and with the intention of simulating more realistic behaviors we plan to add a market situation awareness system to the available services so they can really compete with each other for winning bids and ultimately resulting in better price services for CADOs. Currently, CADOs goal is to be accessible at the end of the simulated period but, on the contrary, preservation services do not have a goal in the simulator. Future updates will add clear objectives for the services like, for example, maximizing benefits or minimizing total operations cost at the end of the simulation.

With respect to the TiM simulator, an extensive upgrade is currently under development. Authors are working on a new, more ambitious emulation architecture based on blockchain technology and smart contracts. This emulator will allow scalable experimentation with real digital assets, paired with NFTs (non-fungible tokens), with its own associated wallet to store funding for preservation. The distributed blockchain network will host oracle services that enable CADOs (stored digital asset + NFT) to request information 
regarding quality standards and hire preservation services when needed. New intelligent object behaviors, as well as more negotiation protocols will be added to the existing ones inside the new architecture to continue with the research. The new emulation platform will be designed as a completely scalable distributed platform, allowing for nodes (users and offered services) to be added gradually. The new upgrade will multiply the actual simulator capabilities and, at the same time, bring challenges with it, such as security and privacy related issues, which we expect to solve thanks to specific properties of the blockchain technology applied.

Author Contributions: Conceptualization, A.E.-F. and J.L.d.1.R.; data curation, A.E.-F. and J.L.d.l.R.; formal analysis, A.E.-F. and J.L.d.1.R.; funding acquisition, A.E.-F. and J.L.d.1.R.; investigation, A.EF. and J.L.d.1.R.; methodology, A.E.-F. and J.L.d.1.R.; project administration, A.E.-F. and J.L.d.1.R.; resources, A.E.-F. and J.L.d.1.R.; software, A.E.-F. and J.L.d.1.R.; supervision, A.E.-F. and J.L.d.1.R.; validation, A.E.-F. and J.L.d.1.R.; visualization, A.E.-F. and J.L.d.1.R.; writing-original draft, A.E.-F. and J.L.d.l.R.; writing-review and editing, A.E.-F. and J.L.d.1.R. All authors have read and agreed to the published version of the manuscript.

Funding: This research was funded by the PRESERVA 2019 PROD 00024 and VoteVote DEMOC00001 of the AGAUR.

Institutional Review Board Statement: Not applicable.

Informed Consent Statement: Not applicable.

Data Availability Statement: Not applicable.

Conflicts of Interest: The authors declare no conflict of interest.

$\begin{array}{ll}\text { Abbreviations } \\ \text { The following abbreviations are used in this manuscrition } \\ \text { LTDP } & \text { Long-Term Digital Preservation } \\ \text { DP } & \text { Digital Preservation } \\ \text { NFT } & \text { Non-Fungible Token } \\ \text { IDC } & \text { International Data Corporation } \\ \text { SPDO } & \text { Self-Preserving Digital Object } \\ \text { CADO } & \text { Cost-Aware Digital Objec } \\ \text { DO } & \text { Digital Object } \\ \text { TiM } & \text { Simulation time machine } \\ \text { SAW } & \text { Software adoption wave } \\ \text { P } & \text { PRESERVA } \\ \text { mP } & \text { miliPRESERVA } \\ \text { SOA } & \text { Service Oriented Architecture } \\ \text { GMV } & \text { Gross Merchandise Volume } \\ \text { B2B } & \text { Business to Business } \\ \text { B2C } & \text { Business to Consumer } \\ \text { C2C } & \text { Consumer to Consumer } \\ \text { DPB } & \text { Direct Purchase Baseline } \\ \text { RMDA } & \text { Reverse Multi-unit Descending Auction } \\ \text { CDA } & \text { Combinatorial Descending Auction } \\ \text { MAS } & \text { Multi-agent systems } \\ & \end{array}$

\section{References}

1. Sarkissian, J. (CSIRO Parkes Observatory, Parkes, NSW, Australia). The Search for the Apollo 11 SSTV Tapes. Available online: https://honeysucklecreek.net/Apollo_11/tapes/Search_for_SSTV_Tapes.pdf (accessed on 21 October 2021).

2. Risk of Loss of Digital Data and the Reasons It Occurs: Case Studies and Analysis. Digital Preservation Coalition. Available online: http:/ / artsdocbox.com/Television / 67049496-Risk-of-loss-of-digital-data-and-the-reasons-it-occurs-case-studiesand-analysis.html (accessed on 21 October 2021).

3. Lopes, H.; Pires, I.M.; Sánchez San Blas, H.; García-Ovejero, R.; Leithardt, V. PriADA: Management and Adaptation of Information Based on Data Privacy in Public Environments. Computers 2020, 9, 77. [CrossRef] 
4. PLANETS Project. Available online: https:/ / planets-project.eu/ (accessed on 13 October 2021).

5. The Digital Universe in 2020: Big Data, Bigger Digital Shadows, and Biggest Growth in the Far East. Available online: https: //www.speicherguide.de/download/dokus/IDC-Digital-Universe-Studie-iView-11.12.pdf (accessed on 13 October 2021).

6. Library of Congress. Available online: https://www.digitalpreservation.gov/ (accessed on 13 October 2021).

7. Netpreserve. Available online: https://netpreserve.org/ (accessed on 13 October 2021).

8. Rothenberg, J. Ensuring the Longevity of Digital Information. Int. J. Leg. Inf. 1998, 26, 1-22. [CrossRef]

9. Digital Preservation Coalition. Digital Preservation Handbook. Available online: https://www.dpconline.org/docs/digitalpreservation-handbook2/1552-dp-handbook-digital-preservation-briefing/file (accessed on 13 October 2021).

10. Olvera, J.A. Digital Preservation: A New Approach from Computational Intelligence. Bull. IEEE Tech. Comm. Digit. Libr. 2013, 9, 22-26. Available online: https:/ / bulletin.jcdl.org/Bulletin/v9n2/papers/olvera.pdf (accessed on 5 October 2021).

11. Cartledge, C.L.; Nelson, M.L. When Should I Make Preservation Copies of Myself? Digit. Libr. 2014, 109-118. [CrossRef]

12. De la Rosa, J.L.; Olvera, J.A. First Studies on Self-Preserving Digital Objects. In Proceedings of the 15th International Conference of the Catalan Association of Artificial Intelligence, Alacant, Spain, 25-26 October 2012; pp. 213-222. [CrossRef]

13. Nelson, M. Buckets: Smart Objects for Digital Libraries. Ph.D. Thesis, Old Dominion University, Norfolk, VI, USA, 2001. [CrossRef]

14. De la Rosa, J.L.; Hormazábal, N.; Aciar, S.; Lopardo, G.; Trias, A.; Montaner, M. A Negotiation Style Recommender Based on Computational Ecology in Open Negotiation Environments. IEEE Trans. Ind. Electron. 2011, 58, 2073-2085. [CrossRef]

15. El-Fakdi, A.; De la Rosa, J.L. Analysis of Nature-Inspired Algorithms for Long-Term Digital Preservation. Mathematics 2021 9, 2279. [CrossRef]

16. Lomuscio, A.R.; Wooldridge, M.; Jennings, N.R. A Classification Scheme for Negotiation in Electronic Commerce. In Agent Mediated Electronic Commerce. Lecture Notes in Computer Science; Dignum, F., Sierra, C., Eds.; Springer: Berlin/Heidelberg, Germany, 2001; Volume 1991, pp. 19-33. [CrossRef]

17. Giaretta, D. Introduction to OAIS Concepts and Terminology. In Advanced Digital Preservation; Springer: Berlin/Heidelberg, Germany, 2011; pp. 13-30. [CrossRef]

18. Weibel, S.; Kunze, J.; Lagoze, C.; Wolf, M. Dublin Core Metadata for Resource Discovery. RFC 1998, 2413, 132. [CrossRef]

19. IFLA, Networking for Digital Preservation: Current Practice in 15 National Libraries. Available online: https:/ /www.ifla.org/ ES/publications/ifla-publications-series-119 (accessed on 18 October 2021).

20. UNESCO: Digital Preservation Programme. Available online: https://en.unesco.org/themes/information-preservation/digitalheritage/digital-preservation-programmes (accessed on 18 October 2021).

21. Ramalho, J.; Ferreira, M.; Faria, L.; Castro, R.; Barbedo, F.; Corujo, L. RODA and CRiB a Service-Oriented Digital Repository. In Proceedings of the Fifth International Conference on Preservation of Digital Objects, London, UK, 29-30 September 2008. Available online: http:/ / repositorium.uminho.pt/bitstream/1822/8226/1/RodaAndCrib.pdf (accessed on 18 October 2021).

22. Smith, M.; Bass, M.; McClellan, G.; Tansley, R.; Barton, M.; Branschofsky, M.; Stuve, D.; Walker, J.H. DSpace: An Open Source Dynamic Digital Repository. D-Lib Mag. 2003, 9. [CrossRef]

23. Daniel, R.; Lagoze, C. Distributed active relationships in the Warwick framework. In Proceedings of the Second IEEE Metadata Workshop, Silver Spring, MD, USA, 16-17 September 1997. Available online: http:/ / citeseerx.ist.psu.edu/viewdoc/download? doi=10.1.1.50.1685\&rep=rep1\&type=pdf (accessed on 18 October 2021).

24. Beazley, M.R. Eprints Institutional Repository Software: A Review. Partnersh. Can. J. Libr. Inf. Pract. Res. 2011, 5. [CrossRef]

25. Filecoin Is a Decentralized Storage Network Designed to Store Humanity's Most Important Information. Available online: https: / filecoin.io/ (accessed on 14 October 2021).

26. Storj: Decentralized Cloud Storage. Available online: https://www.storj.io/ (accessed on 14 October 2021).

27. Sia: Decentralized Storage for the Post-Cloud World. Available online: https://sia.tech/ (accessed on 14 October 2021).

28. Chapman, S. Counting the Costs of Digital Preservation: Is Repository Storage Affordable? J. Digit. Inf. Econ. Factors Digit. Libr. 2004, 2. Available online: https://journals.tdl.org/jodi/index.php/jodi/article/view/jodi-113 (accessed on 5 October 2021).

29. Rusbridge, C. Excuse Me...Some Digital Preservation Fallacies? Ariadne Web Mag. Inf. Prof. 2006, 46. Available online: http:/ / www.ariadne.ac.uk/issue/46/rusbridge/ (accessed on 5 October 2021).

30. Strodl, S.; Rauber, A.; Rauch, C.; Hofman, H.; Debole, F.; Amato, G. The DELOS testbed for choosing a digital preservation strategy. In Proceedings of the Digital Libraries: Achievements, Challenges and Opportunities, 9th International Conference on Asian Digital Libraries, ICADL, Kyoto, Japan, 27-30 November 2006; pp. 323-332. [CrossRef]

31. Currall, J.; Mckinney, P. Investing in Value: A Perspective on Digital Preservation. D-Lib Mag. 2006, 12. Available online: https://www.dlib.org/dlib/april06/mckinney/04mckinney.html (accessed on 5 October 2021). [CrossRef]

32. Life Project. Available online: http://www.life.ac.uk/ (accessed on 6 October 2021).

33. Berman, F.; Lavoie, B.; Ayris, P.; Choudhury, G.S.; Cohen, E.; Courant, P.; Dirks, L.; Friedlander, A.; Gurbaxani, V.; Jones, A.; et al. Sustainable Economics for a Digital Planet: Ensuring Long-Term Access to Digital Information; Final Report of the Blue Ribbon Task Force on Sustainable Digital Preservation and Access; Rensselaer Polytechnic Institute: Troy, NY, USA, 2010. Available online: https: / / discovery.ucl.ac.uk/id/eprint/19116/ (accessed on 5 October 2021).

34. KRDS/I2S2 Digital Preservation Benefit Analysis Tools Project. Available online: https://beagrie.com/krds-i2s2.php (accessed on 6 October 2021). 
35. Currall, J.; McKinney, P.; Johnson, C. Digital Preservation as an Albatross. In Proceedings of the Archiving, Ottawa, ON, Canada, 23-26 May 2006. Available online: http:/ / eprints.gla.ac.uk/140507/ (accessed on 5 October 2021).

36. Olvera, J.A.; Carrillo, P.; De la Rosa, J.L. Combinatorial and Multi-Unit Auctions Applied to Digital Preservation of Self-Preserving Objects. In Proceedings of the Artificial Intelligence Research and Development-Recent Advances and Applications, Barcelona, Catalonia, Spain, 22-24 October 2014; Cabedo, L.M., Pujol, O., Agell, N., Eds.; IOS Press: Amsterdam, The Netherlands, 2014; Volume 269, pp. 265-268. [CrossRef]

37. Garret, J.; Waters, D. Preserving Digital Information: Report of the Task Force on Archiving of Digital Information; The Commission on Preservation and Access and The Research Libraries Group Inc.: Washington, DC, USA, 1996; ISBN 978-1887334501.

38. Rothenberg, J. Avoiding Technological Quicksand: Finding a Viable Technical Foundation for Digital Preservation, 1st ed.; Council on Library and Information Resources: Arlington, VA, USA, 1999; ISBN 1-887334-63-7.

39. Ruusalepp, R.; Dobreva, M. Digital Preservation Services: State of the Art Analysis. In Research Report from Collection Scholarly Works_FacMKSLIAS, Funded by the DC-NET Project; University of Malta: Valletta, Malta, 2012. Available online: https://www. um.edu.mt/library/oar/handle/123456789/311 (accessed on 5 October 2021).

40. Abrams, S.; Kunze, J.; Loy, D. An Emergent Micro-Services Approach to digital Curation Infrastructure. Int. J. Digit. Curation 2010, 5, 172-186. [CrossRef]

41. Xu, H.; Russell, T.; Coposky, J.; Rajasekar, A.; Moore, R.; de Torcy, A.; Wan, M.; Shroeder, W.; Chen, S. iRODS Primer 2: Integrated Rule-Oriented Data System. In Synthesis Lectures on Information Concepts, Retrieval, and Services; Marchionini, G., Ed.; Morgan \& Claypool Publishers: Williston, VT, USA, 2017; Volume 9, pp. 1-131. [CrossRef]

42. Kurhinen, H.; Lampi, M. A Community Driven Micro-Services Architecture Supporting Long Term Digital Preservation. In Proceedings of the Archiving Conference, Berlin, Germany, 13-16 May 2014; Society for Imaging Science and Technology: Springfield, VA, USA, 2014; Volume 5, pp. 47-51. Available online: https:/ /www.ingentaconnect.com/content/ist/ac/2011/000 02011/00000001/art00026 (accessed on 1 November 2021).

43. Archivematica. Available online: https://www.archivematica.org/en/ (accessed on 1 November 2021).

44. OnSale. Available online: https://www.nytimes.com/1995/05/23/business/company-news-auction-of-collectibles-on-theinternet.html (accessed on 4 November 2021).

45. eBay. Available online: https://www.ebay.com/ (accessed on 4 November 2021).

46. eBay Results 2020. Available online: https://investors.ebayinc.com/investor-news/press-release-details/2021/eBay-Inc. -Reports-Better-Than-Expected-Fourth-Quarter-and-Full-Year-2020-Results/default.aspx (accessed on 4 November 2021).

47. Dobzinski, S.; Nisan, N. Mechanisms for Multi-Unit Auctions. J. Artif. Intell. Res. 2010, 37, 85-98. [CrossRef]

48. Tenorio, R. Revenue Equivalence and Bidding Behavior in a Multi-Unit Auction Market: An Empirical Analysis. Rev. Econ. Stat. 1993, 75, 302-314. [CrossRef]

49. Adam, M.; Eidels, A.; Lux, E.; Teubner, T. Bidding Behavior in Dutch Auctions: Insights from a Structured Literature Review. Int. J. Electron. Commer. 2017, 21, 363-397. [CrossRef]

50. Cramton, P.; Shoham, Y.; Steinberg, R. Combinatorial Auctions; MIT Press: Cambridge, MA, USA, 2006; ISBN 9780262033428. [CrossRef]

51. Olvera, J.A.; de la Rosa, J.L. Time Machine: Projecting the Digital Assets onto the Future Simulation Environment. In Advances in Practical Applications of Agents, Multi-Agent Systems, and Sustainability: The PAAMS Collection, Proceedings of the PAAMS 2015, Salamanca, Spain, 3-4 June 2015; Demazeau, Y., Decker, K., Bajo Pérez, J., de la Prieta, F., Eds.; Lecture Notes in Computer Science; Springer: Cham, Switzerland, 2015; Volume 9086, pp. 175-186. [CrossRef] 